This research is published in its final and definitive form by Springer in the journal Psychopharmacology. Please consult this journal for a final, authoritative version. The original publication is available at www.springerlink.com

\title{
Glucose effects on long-term memory performance: duration and domain specificity.
}

Lauren Owen ${ }^{1(\bowtie)}$, Yvonne Finnegan ${ }^{2}$, Henglong $\mathrm{Hu}^{2}$, Andrew B Scholey ${ }^{1}$, Sandra I. Sünram-Lea ${ }^{3}$

${ }^{1}$ NICM Centre for the Study of Natural Medicines and Neurocognition, Brain Sciences Institute, Melbourne, VIC 3122, Australia.

${ }^{2}$ Royal Forest Factory, Coleford, Gloucestershire, GL16 8JB, UK

${ }^{3}$ Department of Psychology, University of Lancaster, Lancaster LA1 4YW, UK

$(\bowtie)$ To whom correspondence should be addressed;

Dr. Lauren Owen, NICM Centre for Neurocognition, Brain Sciences Institute, Melbourne, VIC 3122, Australia.

$\mathrm{T}: \quad+61392144477$

F: $\quad+61392145525$

E-mail: lauren.j.owen@gmail.com

\section{Acknowledgements}

GlaxoSmithKline (GSK) funded this project as part of a PhD program. 


\begin{abstract}
Rational; Previous research has suggested that long term- verbal declarative memory is particularly sensitive to enhancement by glucose loading, however investigation of glucose effects on certain memory domains has hitherto been neglected. Therefore domain specificity of glucose effects merits further elucidation.

Objectives; The aim of the present research was to provide a more comprehensive investigation of the possible effects of glucose administration on different aspects of memory by i) contrasting the effect of glucose administration on different memory domains (implicit/ explicit memory; verbal/ non-verbal memory, recognition/ familiarity processes), ii) investigating whether potential effects on memory domains differ depending on the dose of glucose administered (25g versus $60 \mathrm{~g}$ ), iii) exploring the duration of the glucose facilitation effect (assessment of memory performance 35 min and 1 week after encoding).

Methods; a double blind, between- subjects design was used to test the effects of administration of 25 and $60 \mathrm{~g}$ glucose on memory performance.

Results; Implicit memory was improved following administration of $60 \mathrm{~g}$ of glucose. Glucose supplementation failed to improve face recognition performance but significantly improved performance of word recall and recognition following administration of $60 \mathrm{~g}$ of glucose. However, effects were not maintained one-week following encoding.
\end{abstract}

Conclusions; Improved implicit memory performance following glucose administration has not been reported before. Furthermore the current data tentatively suggest that level of processing may determine the required glucose dosage to demonstrate memory improvement and that higher dosages may be able to exert effects on memory pertaining to both hippocampal and non-hippocampal brain regions. 
Keywords: Glucose, implicit memory, face recognition, long-term memory.

\section{Introduction}

Previous work has demonstrated that glucose administration reliably improves verbal long-term memory performance (for reviews see Hoyland et al. 2008; Messier 2004; Riby 2004). Since glucose is the primary substrate for brain metabolism the delineation of any differential glucose effects on memory might help the understanding of fundamental brain-behaviour relationships - for example whether certain memory functions are prioritised or limited by glucose availability. Additionally, glucose enhancement of memory offers a useful prototype to develop paradigms for studying the effects of more complex nutrition-like interventions. Poor glucose metabolism is also observed in a number of pathologies and thus may afford some insight into the memory problems that accompany these disease states. The fractionation of the glucose enhancement effect has previously been attributed to the degree to which memory tasks rely on medial temporal structures most strongly associated with declarative memory, such as the hippocampus. The suggestion that glucose administration and/or impairments in glucoregulatory mechanisms exert the most profound effects on medial temporal regions is supported by functional characteristics associated with these areas such as high density of insulin receptors in the hippocampus (e.g. see Messier 2004) which are known to promote cellular glucose uptake (e.g. see Watson and Craft 2008). Insulin-sensitive glucose transporters such as GLUT4 (which mediate passive diffusion of glucose through the blood brain barrier) are also enriched in the hippocampus (though the highest concentration is in the cerebellum, see McEwen and Reagan, 2004).

In terms of declarative memory, much of the previous research has focused on the effects of glucose administration on conscious recollection for verbal, spatial and numeric material. In normal human adults explicit verbal recollection is associated with medial temporal and hippocampal function (e.g Nayberg et al, 1996), typical spatial working memory tasks activate right-hemisphere prefrontal, occipital, parietal and premotor cortices (Jonides et al, 1993). Another example of declarative or explicit memory is our ability to remember and recognize faces. Face recognition memory 
processes involve small regions of the left and right fusiform and inferior temporal gyri (e.g. Allison, et al 1994). To date, only few studies have examined the effects of glucose administration on our ability to recognize faces. Metzger (2000) investigated the effect of glucose consumption on a face recognition task. Using a population of healthy young adults, glucose $(50 \mathrm{~g})$ or saccharin administration took place 15 min prior to the face recognition task. They observed no differences between glucose and placebo on the faces hit rate (target identification). However, further analyses revealed that participants who had consumed glucose committed significantly fewer false alarms and had higher d-prime scores (a signal detection measure of recognition efficiency) compared with participants who consumed saccharin prior to the test, though this effect did not reach significance. In a later study, Metzger and Flint (2003) found that following administration of $50 \mathrm{~g}$ of glucose participants recognized more target faces than subjects who consumed the placebo drink (saccharin). These findings suggest that face recognition performance might be sensitive to glucose administration.

Long-term memory is not a unitary function but instead encompasses a variety of dissociable processes believed to be mediated by distinct brain systems. For example, long-term memory may be divided into explicit or implicit memories and may be further categorised based on types of information that are stored for example visual, spatial, verbal or auditory types of information. Clear substantiation of the specific domains of long-term memory affected by glucose administration remains to be explored. For example, although previous studies have utilized a variety of procedures and paradigms, to date there is only one published study which investigated the effect of glucose administration $(50 \mathrm{~g})$ on non-conscious or implicit memory. Manning et al. (1997) found no effect of glucose administration on both aspects of memory (implicit and explicit) in healthy young adults. In older adults explicit memory performance was improved, whereas no effect was observed on a measure of implicit memory in that population.

Declarative memory can be further discriminated by memory retrieval processes (either by recognition or familiarity processes). Research suggests that the hippocampal region is critical for recollection, whereas surrounding structures in the medial and inferior temporal lobe (e.g., the parahippocampal gyrus) are important for 
familiarity processes (Aggleton and Brown 1999; Eichenbaum et al. 1994). Recent research has shown that the proportion of 'remember' responses is significantly greater following glucose administration (Sünram-Lea et al. 2008). Therefore in order to further discern the domain specific characteristics that may underpin the glucose facilitation effect we employed the remember/know paradigm which allows discrimination between remember and familiarity processes at retrieval for the face recognition task.

Previous research has demonstrated that when glucose is administered prior to an encoding event long-term memory facilitation is observed following a short delay (30 minutes) and 24 hours after glucose administration (Sünram-Lea et al. 2002a). Influential models of forgetting (Ebbinghaus, 1885) have argued that, unlike learning which shows a linear relationship between time spent learning and amount learned, the rate of forgetting is non-linear, with the amount of forgetting being initially rapid and then slowing down, in a function that is approximately logarithmic. The majority of forgetting occurs in the first 24 hours and natural memory decay should not be substantially different between a 24 hour period and a 1 week period. If glucose enhances memory during the initial test session we would predict that enhancement should also be observed after a one-week delay. Furthermore it remains to be seen whether the duration of enhancement effects differ depending on memory domain. Consequently, in this study participants' long-term memory performance was tested on the day of glucose administration and 1 week after glucose administration and encoding of the material.

Two glucose dosages were selected for test: $25 \mathrm{~g}$ and $60 \mathrm{~g}$ of glucose. The $25 \mathrm{~g}$ glucose dosage was selected on the basis that our previous work demonstrated cognitive enhancement on a number of measures including verbal recall and recognition and spatial working memory (Sünram- Lea, Owen et al., 2010). Moreover, we have previously observed memory enhancement following administration of $60 \mathrm{~g}$ of glucose (Sünram- Lea, Owen et al, 2010) and therefore the higher dosage of $60 \mathrm{~g}$ glucose was also selected for test.

In light of the few studies investigating the effect of glucose administration on nonverbal (face recognition) and implicit memory tasks, and the fact that none of 
these studies investigated the effects of $25 \mathrm{~g}$ and $60 \mathrm{~g}$ of glucose on these aspects of memory we investigated these important aspects of memory in a population of healthy young adults. Taken previous reports of robust effects on explicit memory, it is now necessary to established whether this effect is also evident on other aspects of memory performance. Thus, the aim of the present research was to provide a more comprehensive investigation of the possible effects of glucose administration on different aspects of long term memory by 1) contrasting the effect of glucose administration on different memory domains (implicit versus explicit memory; verbal versus non-verbal memory, recognition versus familiarity processes), 2) investigating whether potential effects on those memory domains differ depending on the dose of glucose administered (25g versus $60 \mathrm{~g}$ ), and finally 3 ) exploring the duration of any glucose facilitation effect (assessment of memory performance $35 \mathrm{~min}$ and 1 week after encoding).

\section{Method}




\section{Study population}

Ninety undergraduate students (29 males, 61 females) from the University of Lancaster participated in the present experiment for which they were paid $£ 7$. Participants age ranged between 18-30 years (mean age $=21$ years). Participants were not diabetic and had a mean BMI $=22.6 \mathrm{~kg} / \mathrm{m}^{2}$. The study was approved by the Department of Psychology Ethics Committee at Lancaster University, and was conducted in accordance with the Declaration of Helsinki. Written informed consent was obtained from each participant prior to participation in the study.

\section{Study Design and Conditions}

The study followed a between-participant, double-blind, placebo controlled design. A between participant design was necessary due to the assessment of implicit learning and memory. Ninety healthy young adult volunteers were randomly allocated to one of three experimental conditions (with 30 participants per cell) corresponding to $60 \mathrm{~g}$ glucose, $25 \mathrm{~g}$ of glucose, $0 \mathrm{~g}$ of glucose (placebo). Drink administration was doubleblind and all drinks were matched for sweetness.

\section{Treatment}

Drinks were manufactured by GlaxoSmithKline, Coleford, UK. Drinks were 330ml of pink, non-carbonated solutions flavoured with fruit flavourings. Thirty of the drink solutions contained $0 \mathrm{~g}$ glucose, thirty contained $25 \mathrm{~g}$ glucose, and thirty contained $60 \mathrm{~g}$ of glucose. Carbohydrate was provided as glucose syrup. Sweetness and flavour was matched using artificial sweeteners and pharmacologically inactive colourings and flavourings.

\section{Blood glucose measurement}

Blood glucose readings were obtained using the ExacTech ${ }^{\circledR}$ blood glucose monitoring equipment (supplied by MediSense Britain Ltd, 16/17 The Courtyard, Gorsey Lane, Coleshill, Birmingham B46 1JA), following the MediSense recommended procedure.

\section{Procedure}


Participants were recruited using an on-line Research Participation System (Sona Systems Ltd.) run and administered by the Department of Psychology, Lancaster University. The software allows confidential pre-screening of participants (i.e. information regarding exclusion criteria is provided and only participants who are eligible are allowed to sign up for participation). Participants were excluded from taking part if i) they had diabetes mellitus; ii) they were older than 30 years; iii) their first language was not English; and iv) their body mass index (BMI) was greater than 24.9. Each participant was required to attend the laboratory on two occasions. All participants were instructed to fast for 12 hours prior to the first test session (i.e. no food or drink except water before testing) and to refrain from smoking for six hours prior to testing. Upon arrival at the laboratory participants were asked whether they had complied with the restrictions. No dietary or other restrictions were implemented at the second test session. Participants were randomly allocated to a treatment regime (drink) and tested between 9am and 11am.

Upon entering the laboratory participants were shown a list of ingredients for the products they were to consume. Participants then gave written consent and were allocated to a treatment regime. Height, weight and blood glucose levels were then measured. The first study day consisted of a pre-dose and post-dose cognitive testing sessions. Following baseline testing and drink administration participants sat quietly for 15 minutes (in order to ensure successful absorption). With the exception of the semantic priming task, both test sessions (pre- and post-dose testing) comprised completion of parallel versions of the same memory tasks (all tasks are described below). One week following testing, participants returned to the lab and were asked to recall the information learned in the post-dose phase of the initial test session. No fasting restrictions were implemented at the 1-week follow up test session.

\section{Memory tasks}

For both word presentation and face recognition task, presentation of stimuli was computerised using Apple Macintosh computer and displayed on colour monitors. Responses were recorded via keyboard with defined keys for responses. The implicit memory task and word recognition task were presented on paper and responses were 
given via paper and pencil. The experimenter was present during the test sessions. Memory tasks were presented in the following order:

Word presentation: A list of 20 words matched for frequency, concreteness and imagery was presented on the monitor at the rate of 1 every 2 seconds (with an inter-stimulus interval of 1 second) for participants to remember. While participants were presented the 20-item word list, they were required to perform two types of complex hand motor sequences, which were practised with each participant before the first presentation of the word list. Participants were instructed to share their attention equally between the two tasks, and were told that they should perform to the best of their ability on each of the two tasks. There were two different motor sequences. Each motor sequence was performed synchronously with both hands. Sequence one comprises 'fist''chop'-'slap'. Sequence two consists of 'back-slap'-'chop'-'fist'. Each participant is instructed to complete one sequence of movements between successive words on the list. Participants were also instructed to change between the two sequences every fifth word; i.e. sequence $1=$ words $1-5$, sequence $2=$ words $6-10$, sequence $1=$ words $11-15$ and sequence 2 = words 16-20. Participants were not told the number of words in the list, just instructed to change between sequences every fifth word. Additionally, participants were informed that they would not be told when they should change motor sequence, but should themselves keep track of the number of words that had been presented. Participants were instructed to remember as many words as they could from the word list whilst carrying out the hand-movement task. As in our previous studies, no rank of importance between the two tasks was communicated to the participant. The ability of participants to perform the hand-movement task was not assessed and incorrect handmovements were not recorded.

Immediate word recall: Assessed immediate free recall memory performance of supra-span word list. Participants were given 60 seconds to write down as many of the words as possible. Recall was scored for number of correct responses and number of errors.

Face presentation: 40 study items (20 male and 20 female faces) were presented. Each item appeared on the screen for $2 \mathrm{~s}$, with an inter-stimulus interval of 1s. Participants were instructed that they should try to remember the faces, as they would be given a recognition test after a brief interval. 
Implicit memory task: Participants underwent a semantic priming task (McGeorge and Burton 1990) in which they were asked to perform arithmetic computations on 20 strings of four digit numbers (all presented on one sheet of A4 paper). The mathematic computation which they are asked to perform is simply to calculate the sum of the first two digits and then the sum of the last two digits. If the sum of the first two was higher than the sum of the last two digits the participant was required to place a tick in an adjacent box if it was lower than the sum of the last two the participant was required to place a cross in the box e.g. if the participant was shown the number 5639 the calculation would be $5+6=11$ and $3+9=12$ therefore the correct response would be to place a cross in the box. Unbeknownst to participants, every string contained the number 3 . For the subsequent test phase, participants had to indicate which of two displayed strings had previously been presented. In fact, both strings of each pair were new, but one of them contained the number 3 , while the other did not. If participants preferentially selected the strings containing the number 3 (positives), without being conscious of this regularity it was interpreted that implicit priming had occurred. Implicit priming was calculated by number of positives selected at test. Above chance performance indicated that implicit priming had occurred.

Delayed word recall: Assessed delayed free recall memory performance of supra-span word list. Participants were given 60 seconds to write down as many of the words as possible. The task was scored as number correct and errors.

Delayed word recognition: Participant were presented a list of 40 words, 20 of the words were from the previously seen 'word presentation' phase at the beginning of the experiment. The other 20 words were non- target distracter words. Participants were asked to indicate (tick words) if they had been shown these words previously. The task was scored by number of correct responses (hits) and false alarms.

Face recognition: Participants were presented with the 40 target faces and an additional 40 distracter faces and were required to make old/new decisions for each face by means of a key press. Following an old response, participants were further required to make a remember/know/guess decision. A 'remember' response was one in which the participant consciously recollected the appearance of that face in the first part of the experiment. A 'know' response was one in which the participant recognize the face because it felt familiar from the first part of the experiment and was recognized purely on the basis of familiarity. For responses that the participant neither 
recollected nor recognized on the basis of familiarity, but which they felt could not definitely be rejected participants had the option of making a 'guess' response. The instructions for the remember/know responses were closely modelled from those by Rajaram (1993). The task was scored as number of hits and false alarms.

\section{Statistical Analysis}

Blood glucose values were examined using a mixed analysis of variance (ANOVA), the between- subjects factor being drink (3 levels; $0 \mathrm{~g}, 25 \mathrm{~g}$, and $60 \mathrm{~g}$ of glucose) and the repeated measures factor being time (i.e. at what point blood glucose was measured). Where significant statistical effects were identified by ANOVA, post-hoc analysis using Bonferroni paired comparisons were subsequently conducted.

For memory measures on testing sessions 1 and 2 (with the exception of the implicit memory task) the scores were transformed into change from baseline scores (post pre). The resulting change from baseline scores were analysed using one -way ANOVAs with drink as the between-subject factor. This was followed by planned (apriori) comparisons using contrast tests between the two experimental groups and the control group (25g and $60 \mathrm{~g}$ of glucose versus Placebo). In addition, where significant statistical effects were identified by ANOVA, post-hoc analysis using Bonferroni paired comparisons were subsequently conducted.

For the data collected 1-week post drink administration, the effect of drink was analysed using one-way ANOVA for absolute performance levels. Further planned comparisons were then carried out to investigate differences in performance for each of the two different glucose drinks (i.e. 25 and $60 \mathrm{~g}$ ).

The implicit memory task was scored by number of positives selected at test which were compared to chance performance (10/20) using t-test.

\section{Result}

Glycaemic response 
There was a main effect of drink $(F(2,87)=47.47, \mathrm{p}<.001)$ and a main effect of time $(\mathrm{F}(3,261)=140.61, \mathrm{p}<0.001)$. There was also a significant time by drink interaction $(\mathrm{F}(6,261)=42.27, \mathrm{p}<0.001)$. Further post hoc analysis demonstrated that although baseline BGLs (T0) did not significantly differ across drinks, following administration of both glucose drinks participants had significantly higher blood glucose levels compared to placebo $(\mathrm{p}<0.001)$. Blood glucose levels were not significantly higher for those in the $60 \mathrm{~g}$ glucose condition compared to those observed after ingestion of $25 \mathrm{~g}$ of glucose 15,35 , and 48 minutes following consumption ( $\mathrm{p}=$ $0.960, p=0.220, p=1.0$, respectively) see Table 1 ).

\section{Table 1 around here}

\section{Memory performance}

For all means and standard deviations and planned comparisons of explicit memory performance see table 2. All significant results are presented in Figure 1.

\section{Table 2 around here}

Immediate free recall: There was a trend towards a significant main effect of drink on immediate free recall $(\mathrm{F}(2,87)=2.648, \mathrm{p}=0.077)$. Planned comparisons revealed significantly improved performance following $60 \mathrm{~g}$ glucose compared to placebo $(p<0.02)$. See figure 1a for immediate recall performance as a function of drink.

Delayed free recall: No main effect of drink was observed on delayed free recall performance $(\mathrm{F}(2,87)=0.580, \mathrm{p}=0.562)$ and none of the planned comparison reached significance.

\section{Figure 1 around here}

Word recognition: There was a significant main effect of drink on word recognition $(\mathrm{F}(2,87)=4.155, \quad \mathrm{p}=0.019)$. Planned comparisons revealed that following administration of $60 \mathrm{~g}$ of glucose participants correctly recognised significantly more words than those in the placebo group $(\mathrm{p}<0.01)$. Comparison of the placebo and $25 \mathrm{~g}$ of glucose showed no significant differences, however following up the significant 
ANOVA result post hoc comparison showed that participants correctly recognised more words following administration of $60 \mathrm{~g}$ of glucose compared to $25 \mathrm{~g}$ of glucose. However, the latter finding just failed to withstand post-hoc Bonferroni correction $(\mathrm{p}=0.067)$ (see figure $1 \mathrm{~b})$. There was also a significant main effect of drink on false alarms (non-studied words falsely recognized) $(\mathrm{F}(2,87)=4.1534, \mathrm{p}<0.044)$. Planned comparisons revealed an increase in false alarms following administration of $25 \mathrm{~g}$ of glucose compared to placebo and $60 \mathrm{~g}$ of glucose (both $\mathrm{p}=0.03$ ).

Face recognition: There was no main effect of drink on face recognition hits $(\mathrm{F}(2,78)$ $=.159, \mathrm{p}=.854)$. Further analysis of the subjective recognition experience (rememberknow procedure) showed that there was no effect of drink on number of 'remember' $(\mathrm{F}(2,78)=1.613, \mathrm{p}=.206)$ or 'know' responses $(\mathrm{F}(2,78)=.242, \mathrm{p}=.786)$. In addition, no significant effect of drink was observed on face recognition reaction time $(\mathrm{F}(2,78)$ $=.782, \mathrm{p}=.461)$. Planned comparisons revealed no significant difference of face recognition hits and reaction time between glucose and placebo groups.

Implicit memory: The mean number of positives selected at test (regardless of drink) was 10.662 (out of a possible 20) with a standard deviation of 2.231 . When each of the drink groups were compared to chance it was revealed that following administration of the placebo drink there was no significant difference between test score and chance performance $(\mathrm{t}(29)=.537, \mathrm{p}=.596)$, nor was there a significant difference of positives selected at test compared to chance following $25 \mathrm{~g}$ glucose $(\mathrm{t}(29)=1.472, \mathrm{p}=.152)$. However following a $60 \mathrm{~g}$ glucose load number of positives selected at test was significantly higher than chance $(\mathrm{t}(29)=2.481, \mathrm{p}=.019)$ (mean $=11.033 \pm 2.281)$ (see figure 1c).

Memory performance following a one-week delay.

There were no significant effects of drink on memory performance following a 1 week delay. Data following a one-week delay are presented as absolute values in Table 2).

\section{Discussion}


The aim of the present study was to assess the effect of glucose administration on different memory domains by investigating the possible dissociation of glucose effects on measures of implicit and explicit memory, and by assessing glucose effects on face recognition. In addition, we aimed to investigate whether potential effects on those memory domains differ depending on the dose of glucose administered $(25 \mathrm{~g}$ versus $60 \mathrm{~g}$ ), and to explore the duration of any memory facilitation effects by assessing memory performance one week after encoding.

In terms of glycaemic response, as expected blood glucose levels were raised significantly in the two glucose conditions following drink consumption. Though blood glucose levels were slightly higher following administration of $60 \mathrm{~g}$ of glucose compared to $25 \mathrm{~g}$ of glucose, this did not reach significance over the three time points. This was slightly unexpected as in previous work (Sünram-Lea, Owen, et al., 2010) we have observed significant differences between these glucose dosages over a similar time scale. However a study conducted by Azari (1991) found that while both $30 \mathrm{~g}$ and $100 \mathrm{~g}$ of glucose administration elevated blood glucose levels significantly compared to placebo, blood glucose levels following both glucose dosages were not significantly different. It is possible that failure to observe significant difference between the two glucose dosages reflect variability in terms of glucoregulatory control within the sample. Variation here may also be somewhat inflated due the necessary use of a between-subjects design.

In terms of explicit/declarative memory performance measures, improvements were observed on immediate word recall and delayed recognition following administration of $60 \mathrm{~g}$ of glucose only. While it is not surprising that glucose administration improved declarative memory performance, the observation that stronger facilitation was observed following $60 \mathrm{~g}$ of glucose as opposed to $25 \mathrm{~g}$ of glucose was unexpected. Interestingly the optimal dose here is different to that in a previous study (SünramLea and Owen et al, 2010) which may reflect a cohort effect. The issue of establishing optimal glucose dosages is under-researched in this area and may depend on individual differences in glucoregulation.

It is also important to note that studies which have reliably shown glucose facilitation of long-term memory performance following $25 \mathrm{~g}$ of glucose have generally used 
repeated exposure to the to-be-remembered material (e.g. Foster et al. 1998; SünramLea et al. 2002a; b). Similarly there are reports showing no differential glucose enhancement of memory following single exposure to the to-be-remembered material following 25g glucose (Scholey et al. 2001; Scholey et al. 2009) or at a slightly higher dosage of $37.5 \mathrm{~g}$ glucose (Scholey and Kennedy, 2004). Practice and repetition aid a learning episode and the memory trace is strengthened through repetition of stimuli. It is interesting to note that when recall was assessed 20minutes following word presentation, glucose facilitation was not observed either in the current study Consequently, a possible interpretation of the present finding may be that in order to reveal a glucose effect of materials more shallowly encoded, a greater glucose load is required.

Regarding memory of non-verbal material, no effect of glucose administration was observed on the face recognition task using the remember/know paradigm. Glucose effects on face recognition have not been widely researched and have demonstrated mixed results. Metzger and Flint (2003) found that following administration of $50 \mathrm{~g}$ of glucose participants recognized more target faces than subjects who consumed the placebo drink (saccharin). Metzger (2000) investigated the effect of glucose consumption on a facial recognition task in young adults, using $50 \mathrm{~g}$ glucose and found no effects of face recognition. However they did observe that following a glucose drink participants made significantly fewer errors. We hypothesised that by further investigating this effect using the remember/know paradigm it may be possible to tease apart some underlying memory processes which glucose may be affecting. We did not observe any meaningful effects of glucose using this paradigm and thus find no support for a glucose facilitation effect on face recognition either by remember or familiarity processes. It is possible that facial processing is particularly stable and robust and thus not as sensitive to dietary changes compared some other memory domains. Or it may also be possible that failure to observe facilitation is due to task factors such as difficulty. If indeed task difficulty is a mediating factor for glucose facilitation then a potentially interesting future study might aim to increase the difficulty of the face recognition task by, for example, including a secondary task at the stage of encoding. 
Implicit priming (learning) was observed after administration of $60 \mathrm{~g}$ of glucose, only. To date, this is the first study that demonstrated glucose facilitation of non-conscious or implicit memory, as Manning et al. (1997) found no effect of glucose administration on implicit memory performance in healthy young and older adults. We hypothesised that glucose effects would be preferentially observed on hippocampally mediated memory systems such as explicit memory. The medial temporal lobe, basal forebrain, and diencephalon support the formation of new explicit memories, but do not appear to contribute to the formation of new implicit memories (Squire and Zola-Morgan 1991). Areas of the brain thought to pertain to tasks such as repetition priming (and the formation of implicit memories) are thought to involve the occipital and posterior temporal cortices (see for example Keane et al. 1991; Tulving and Schacter 1990). The fact that glucose facilitation was observed on measures of implicit memory following administration of $60 \mathrm{~g}$ of glucose, tentatively suggests that higher glucose dosages may be able to exert effects on brain areas other than the medial temporal lobe.

Indeed evidence from the animal literature suggests that different doses of glucose can influence different types of memory. More specifically, research in rats showed that the dose-response curve for the effect of post-training glucose injection seems to be bimodal, as peaks were found both at $100 \mathrm{mg} / \mathrm{kg}$ and $2000 \mathrm{mg} / \mathrm{kg}$ (White 1991). It was suggested that these doses may represent the action of glucose on two brain substrates: the dorsal striatum (caudate nucleus and putamen) and the hippocampus. Research in rodents and mammals (incl. humans) has shown a double dissociation between the mnemonic functions of the hippocampus and the dorsal striatum, with the latter being associated with response learning (habit memory) and the former with 'cognitive' learning (cognitive memory) (Packard et al. 1989; Packard and White 1990). Two radial maze tasks were used in these studies, one of which was blocked by caudate nucleus lesions and which has been associated with response learning (habit memory) (win-stay task), and one which was blocked by fornix lesions and has been associated with the cognitive learning memory (win-shift task). White (1991) demonstrated that hippocampally dependent memory was enhanced by glucose doses at both $2000 \mathrm{mg} / \mathrm{kg}$ and $100 \mathrm{mg} / \mathrm{kg}$. However, enhanced response learning was only observed at the higher dose. The reason for the observed enhancement of implicit memory performance following the higher glucose dosage might be mediated by 
amygdala-hippocampal interaction. Data suggest that emotional arousal activates the amygdala, and that such activation results in the modulation of memory storage occurring in other brain regions (McGaugh, Cahill and Roozendaal, 1996). A series of recent studies has shown that emotional arousal can determine the use of hippocampus-dependent or dorsal striatal dependent learning and memory processes in tasks that can be acquired using either response or cognitive learning (for a recent review see Packard 2009). Taken together the data suggest that at low doses glucose administration either fails to activate amygdala- hippocampal interactions or does so in a manner that favours cognitive learning and memory. At higher dosages (one could argue at a level which is more similar to heightened arousal and/or stress) activation of the basolateral amygdala results in an increase in habit memory. The levels of peripheral glucose elevation observed in human laboratory studies of emotional processing are much lower than those observed here (Scholey et al. 2006). However we do not know the levels of central blood glucose which are reached during real-life stressors, nor the extent to which central changes may differ from those observed in the periphery.

This model could also be used to explain the failure to demonstrate glucose facilitation of declarative memory at the lower dose as level of processing may interact with the facilitating effects of glucose administration in a manner that could influence the dose- response relationship.

The present study also aimed to investigate the duration of the glucose facilitation effect by addressing the question of whether the effects of glucose administration outlast any evidence of elevated blood glucose levels at the time of memory retrieval. Sünram-Lea et al. (2002a) examined long-term memory facilitation and observed that glucose facilitation persists 24 hours after glucose administration. However, the present study did not demonstrate that glucose facilitation effects were maintained 1 week following encoding. While the delay used in the present study (one week) was substantially longer than the delay implemented by Sünram-Lea et al. (24-hours), it is unlikely that the failure to observe glucose enhancement following a one week delay was solely due to time-dependent memory decay. This argument holds even more as no glucose facilitation was observed after a 20 minute delay. As discussed previously, 
the studies by Sünram-Lea et al. (Sunram-Lea et al. 2004; Sünram-Lea et al. 2001; 2002a; b) utilised a memory task in which repeated recall and thus exposure to the stimulus was likely to ensure a deep memory trace. Although there is no consensus on the mechanisms responsible for practice/repetition and forgetting, the failure in this study for the glucose facilitation effect to be observed following a 20minute and a one-week delay could be due to insufficient trace strength which is needed to ensure greater protection against increasing decay and/or increased interference over time.

In summary the finding that explicit and implicit memory was facilitated following administration of $60 \mathrm{~g}$ of glucose was surprising. As mentioned earlier, the current data tentatively suggest that i) higher glucose dosages may be able to exert effects on brain areas other than the medial temporal lobe and ii) that level of encoding could be an additional factor determining optimal dose for enhancement of long-term declarative memory. 


\section{References}

Aggleton J, Brown M (1999) Episodic memory, amnesia, and the hippocampalanterior thalamic axis. Behavioral and Brain Sciences 22:425-444.

Allison T, Ginter H, McCarthy G, Nobre AC, Puce A, Luby M, Spencer DD (1994) Face recognition in human extrastriate cortex. Journal of Neurophysiology, Vol 71, Issue $2821-825$.

Azari N (1991) Effects of glucose on memory processes in young adults. Psychopharmacology 105:521-524.

Ebbinghaus H (1885) Über das Gedächtnis. Leipzig; Dunker (Translation by Ruyer, H., Bussenius, C.E. (1913) Memory. Newyour; Teachers College, Columbia University.

Eichenbaum H, Otto T, Cohen N (1994) Two functional components of the hippocampal memory system. Behavioral and Brain Sciences 17:449-517.

Foster J, Lidder P, Sünram S (1998) Glucose and memory: fractionation of enhancement effects? Psychopharmacology 137:259-270.

Jonides J, Smith EE, Koeppe, RA, Awh E, Minoshima S, Mintun MA (1993) Spatial working-memory in humans as revealed by PET. Nature 363, 623-625

Hoyland A, Lawton C, Dye L (2008) Acute effects of macronutrient manipulations on cognitive test performance in healthy young adults: A systematic research review. Neuroscience and Biobehavioral Reviews 32:72-85.

Keane M, Gabrieli J, Fennema A, Growdon J, Corkin S (1991) Evidence for a dissociation between perceptual and conceptual priming in Alzheimer's disease. Behavioral Neuroscience 105:326-342. 
Manning C, Parsons M, Cotter E, Gold P (1997) Glucose Effects on Declarative and Nondeclarative Memory, in Healthy Elderly and Young Adults. Psychobiology 25:103-108.

McEwen B, Reagan L (2004) Glucose transporter expression in the central nervous system: relationship to synaptic function. European journal of pharmacology 490:1324.

McGaugh J, Cahill L, Roozendaal B (1996) Involvement of the amygdala in memory storage: interaction with other brain systems. Proceedings of the National Academy of Sciences of the United States of America 93:13508.

McGeorge P, Burton A (1990) Semantic processing in an incidental learning task. The Quarterly Journal of Experimental Psychology Section A 42:597-609.

Messier C (2004) Glucose improvement of memory: a review. European journal of pharmacology 490:33-57.

Metzger M (2000) Glucose enhancement of a facial recognition task in young adults. Physiology \& behavior 68:549-553.

Metzger M, Flint R (2003) Glucose enhancement of face recognition is unaffected by alterations of face features. Neurobiology of Learning and Memory 80:172-175.

Nyberg, L., Mclntosh, A.R., Houle, S., Nilsson, L-G., Tulving, E. (1996) Activation of medial temporal structures during episodic memory retrieval. Nature 380, 715-717.

Packard M, Hirsh R, White N (1989) Differential effects of fornix and caudate nucleus lesions on two radial maze tasks: evidence for multiple memory systems. Journal of Neuroscience 9:1465.

Packard M, White N (1990) Lesions of the caudate nucleus selectively impair "reference memory" acquisition in the radial maze. Behav Neural Biol 53:39-50. 
Packard M (2009) Anxiety, cognition, and habit: A multiple memory systems perspective. Brain Research 1293:121-128.

Rajaram S (1993) Remembering and knowing: Two means of access to the personal past. Memory and Cognition 21:89-89.

Riby L (2004) The impact of age and task domain on cognitive performance: a metaanalytic review of the glucose facilitation effect. Brain Impairment 5:145-165.

Scholey A, Harper S, Kennedy D (2001) Cognitive demand and blood glucose. Physiology and Behavior 73:585-592.

Scholey A, Kennedy D (2004) Cognitive and physiological effects of an "energy drink": an evaluation of the whole drink and of glucose, caffeine and herbal flavouring fractions. Psychopharmacology 176:320-330.

Scholey A, Laing S, Kennedy D (2006) Blood glucose changes and memory: Effects of manipulating emotionality and mental effort. Biological Psychology 71:12-19.

Scholey A, Sünram-Lea S, Greer J, Elliott J, Kennedy D (2009) Glucose administration prior to a divided attention task improves tracking performance but not word recognition: evidence against differential memory enhancement? Psychopharmacology 202:549-558.

Squire L, Zola-Morgan S (1991) The medial temporal lobe memory system. Science 253:1380.

Sünram-Lea S, Dewhurst S, Foster J (2008) The effect of glucose administration on the recollection and familiarity components of recognition memory. Biological Psychology 77:69-75. 
Sunram-Lea S, Foster J, Durlach P, Perez C (2004) The influence of fat coadministration on the glucose memory facilitation effect. Nutritional Neuroscience 7:21-32.

Sünram-Lea S, Foster J, Durlach P, Perez C (2001) Glucose facilitation of cognitive performance in healthy young adults: examination of the influence of fast-duration, time of day and pre-consumption plasma glucose levels. Psychopharmacology $157: 46$.

Sünram-Lea S, Foster J, Durlach P, Perez C (2002a) The effect of retrograde and anterograde glucose administration on memory performance in healthy young adults. Behavioural brain research 134:505-516.

Sünram-Lea S, Foster J, Durlach P, Perez C (2002b) Investigation into the significance of task difficulty and divided allocation of resources on the glucose memory facilitation effect. Psychopharmacology 160:387-397.

Sünram-Lea S, Owen L, Finnegan Y, Hu H, (2010) Dose-response investigation into glucose facilitation of memory performance and mood in healthy young adults. Journal of Psychopharmacology. In Press.

Tulving E, Schacter D (1990) Priming and human memory systems. Science 247:301.

Watson G, Craft S (2008) Insulin Resistance Alzheimer's Disease: Pathophysiology and Treatment. Progress in Neurotherapeutics and Neuropsychopharmacology 3:85110.

White N (1991) Peripheral and central memory enhancing actions of glucose.

Peripheral Signalling of the Brain: Role in Neural-Immune Interactions, Learning and Memory. Toronto, Hogrefe and Huber 421-443. 


\section{Figure legends}

Table 1

Means $( \pm \mathrm{SD})$ blood glucose levels $(\mathrm{mmol} / \mathrm{l})$ as a function of glucose dose over time. Significant elevation of blood glucose was observed following both glucose loads compared with placebo $(\dagger, \mathrm{p}<.001$ compared with corresponding placebo value; $\S, \mathrm{p}$ $<.05$ between the $25 \mathrm{~g}$ and $60 \mathrm{~g}$ glucose drinks.

Figure 1

Memory measures showing significant effects of glucose. Graphs show Mean ( \pm SEM) for placebo $(0 \mathrm{~g})$ and glucose $(25 \mathrm{~g}$ and $60 \mathrm{~g})$ for (a) immediate word recall (change from baseline), (b) word recognition accuracy (change from baseline) and (c) implicit memory performance. For (a) and (b) *denotes significant improvement compared with placebo $(\mathrm{p}<.05)$; for $(\mathrm{c}) *$ denotes significant improvement $(\mathrm{p}<.05)$ compared with chance performance (broken line).

\section{Table 2}

Means ( \pm SD) scores for all explicit memory measures as a function of glucose dose. Scores are shown for same day baseline, post-drink and change-from-baseline scores, 1 -week delay scores are also presented where appropriate. Significant differences from placebo are shown in bold $(*, p<.05)$. 
Table 1

\begin{tabular}{ccccc}
\cline { 2 - 4 } & Baseline & 15 $\min$ & $35 \min$ & $48 \min$ \\
\hline Placebo & & & & \\
25g Glucose & $4.673 \pm .819$ & $4.493 \pm .763$ & $4.480 \pm .535$ & $4.560 \pm .575$ \\
60g Glucose & $4.603 \pm .625$ & $\mathbf{6 . 2 4 6} \pm \mathbf{1 . 1 1 0} \dagger$ & $\mathbf{7 . 3 4 6} \pm \mathbf{1 . 2 0 0} \dagger$ & $\mathbf{7 . 2 1 3} \pm \mathbf{1 . 3 7 9} \dagger$ \\
& $4.433 \pm .689$ & $\mathbf{6 . 5 1 0} \pm \mathbf{1 . 1 4 0} \dagger$ & $\mathbf{7 . 8 8 3} \pm \mathbf{1 . 4 8 8} \dagger$ & $\mathbf{7 . 4 6 0} \pm \mathbf{1 . 7 9 9} \dagger$ \\
\hline
\end{tabular}


Figure 2
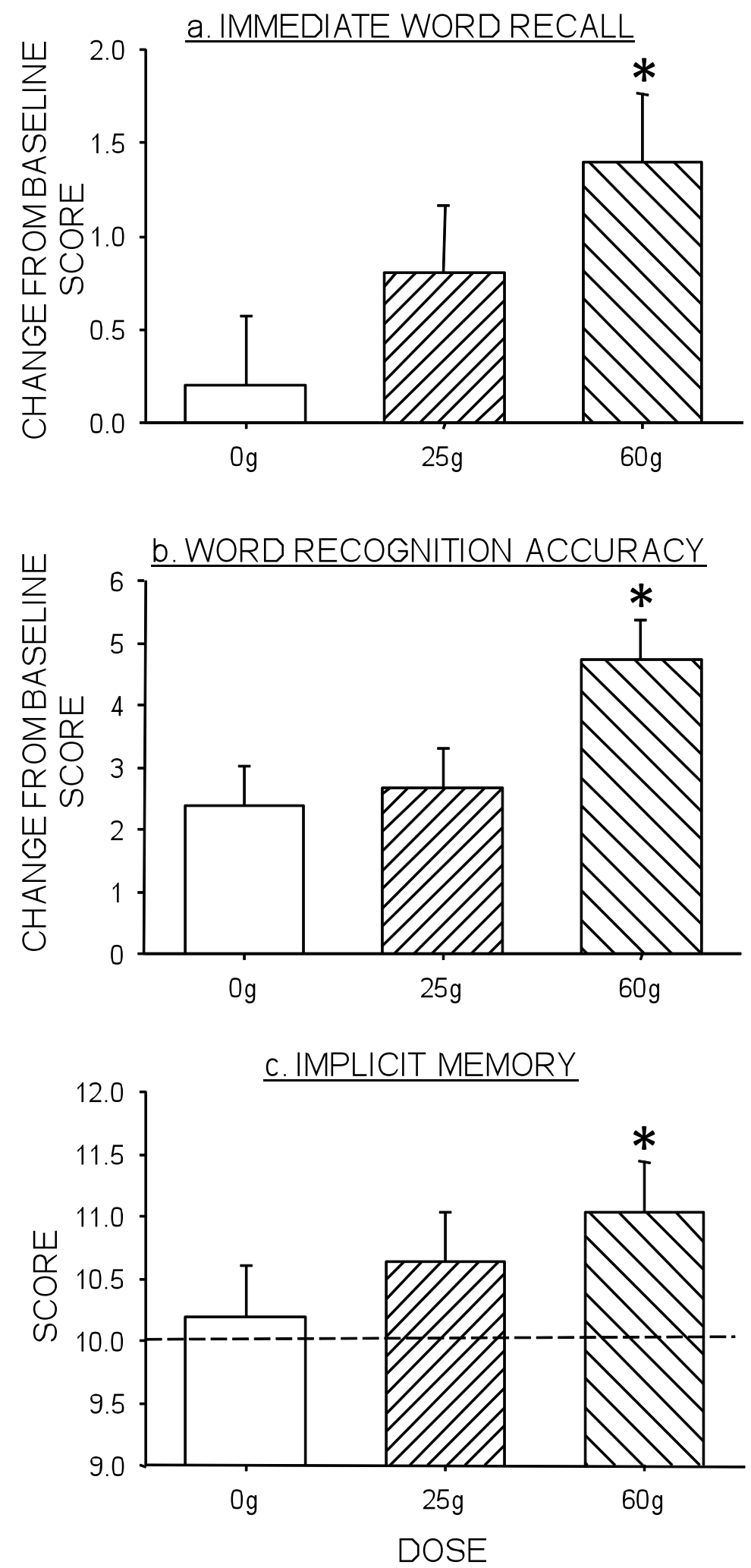
Table 2

\begin{tabular}{|c|c|c|c|c|}
\hline & \multicolumn{3}{|c|}{ Same day testing } & \multirow[b]{2}{*}{ One-week delay } \\
\hline & \multirow[t]{2}{*}{ Baseline score } & \multirow[t]{2}{*}{$\begin{array}{l}\text { Post-drink } \\
\text { score }\end{array}$} & $\begin{array}{l}\text { Change from } \\
\text { baseline score }\end{array}$ & \\
\hline \multicolumn{3}{|c|}{ Immediate word recall (correct) } & & \\
\hline Placebo & $5.533 \pm 1.925$ & $5.740 \pm 1.452$ & $.207 \pm 2.170$ & - \\
\hline $25 \mathrm{~g}$ Glucose & $5.566 \pm 2.223$ & $6.366 \pm 2.311$ & $.800 \pm 1.669$ & - \\
\hline $60 \mathrm{~g}$ Glucose & $5.103 \pm 1.561$ & $6.500 \pm 1.943$ & $1.396 \pm 2.126 *$ & - \\
\hline \multicolumn{5}{|c|}{ Immediate Word Recall (errors) } \\
\hline Placebo & $.750 \pm .771$ & $.566 \pm .678$ & $-.183 \pm 1.027$ & - \\
\hline $25 \mathrm{~g}$ Glucose & $.500 \pm .707$ & $.692 \pm .509$ & $.192 \pm .780$ & - \\
\hline $60 \mathrm{~g}$ Glucose & $.482 \pm .622$ & $.533 \pm .681$ & $.050 \pm .932$ & - \\
\hline \multicolumn{5}{|c|}{ Delayed Recall (correct) } \\
\hline Placebo & $4.880 \pm 1.397$ & $5.035 \pm 1.449$ & $.155 \pm 1.486$ & $2.133 \pm 1.655$ \\
\hline $25 \mathrm{~g}$ Glucose & $4.880 \pm 1.492$ & $5.400 \pm 2.444$ & $.520 \pm 1.984$ & $2.770 \pm 1.739$ \\
\hline $60 \mathrm{~g}$ Glucose & $4.689 \pm 1.821$ & $4.678 \pm 1.682$ & $-.011 \pm 2.302$ & $2.863 \pm 2.416$ \\
\hline \multicolumn{5}{|c|}{ Delayed Recall (errors) } \\
\hline Placebo & $3.333 \pm 2.406$ & $5.035 \pm 1.449$ & $-2.933 \pm 2.323$ & $1.733 \pm 2.016$ \\
\hline $25 \mathrm{~g}$ Glucose & $2.863 \pm 2.359$ & $5.400 \pm 2.443$ & $-2.275 \pm 2.375$ & $1.555 \pm 1.846$ \\
\hline $60 \mathrm{~g}$ Glucose & $2.620 \pm 2.187$ & $4.678 \pm 1.682$ & $-2.120 \pm 2.322$ & $1.681 \pm 1.961$ \\
\hline \multicolumn{5}{|c|}{ Word Recognition (hits) } \\
\hline Placebo & $11.392 \pm 2.985$ & $13.785 \pm 2.952$ & $2.392 \pm 3.538$ & $12.133 \pm 4.074$ \\
\hline $25 \mathrm{~g}$ Glucose & $11.500 \pm 2.894$ & $14.166 \pm 3.040$ & $2.666 \pm 3.227$ & $12.592 \pm 3.992$ \\
\hline $60 \mathrm{~g}$ Glucose & $9.758 \pm 3.136$ & $14.500 \pm 2.038$ & $4.741 \pm 3.576 *$ & $11.181 \pm 4.227$ \\
\hline \multicolumn{5}{|c|}{ Word Recognition (false alarms) } \\
\hline Placebo & $4.407 \pm 2.879$ & $3.233 \pm 2.314$ & $-1.174 \pm 2.496$ & $5.066 \pm 3.194$ \\
\hline $25 \mathrm{~g}$ Glucose & $3.115 \pm 2.186$ & $3.724 \pm 3.703$ & $.608 \pm 3.667$ & $5.148 \pm 3.697$ \\
\hline $60 \mathrm{~g}$ Glucose & $3.642 \pm 2.950$ & $2.482 \pm 2.372$ & $-1.160 \pm 3.104$ & $4.545 \pm 3.924$ \\
\hline \multicolumn{5}{|c|}{ Face Recognition (hits) } \\
\hline Placebo & $62.958 \pm 4.496$ & $53.291 \pm 6.682$ & $-9.666 \pm .6322$ & $45.703 \pm 4.330$ \\
\hline $25 \mathrm{~g}$ Glucose & $61.275 \pm 5.993$ & $53.034 \pm 6.889$ & $-8.241 \pm 7.214$ & $43.619 \pm 9.583$ \\
\hline $60 \mathrm{~g}$ Glucose & $62.321 \pm 5.773$ & $52.035 \pm 6.173$ & $-10.285 \pm 5.636$ & $43.750 \pm 10.130$ \\
\hline \multicolumn{5}{|c|}{ Face Recognition (reaction time) } \\
\hline Placebo & $1.498 \pm .278$ & $1.376 \pm .266$ & $-.121 \pm .233$ & $1.291 \pm .270$ \\
\hline $25 \mathrm{~g}$ Glucose & $1.637 \pm .469$ & $1.420 \pm .316$ & $-.217 \pm .407$ & $1.447 \pm .297$ \\
\hline $60 \mathrm{~g}$ Glucose & $1.610 \pm .488$ & $1.478 \pm .490$ & $-.131 \pm .254$ & $1.289 \pm .681$ \\
\hline \multicolumn{5}{|c|}{ Face Recognition ('Remember') } \\
\hline Placebo & $11.750 \pm 7.212$ & $12.583 \pm 7.270$ & $.833 \pm .409$ & $8.814 \pm 6.220$ \\
\hline $25 \mathrm{~g}$ Glucose & $12.206 \pm 6.899$ & $14.137 \pm 7.693$ & $1.931 \pm 5.573$ & $7.904 \pm 7.594$ \\
\hline $60 \mathrm{~g}$ Glucose & $11.821 \pm 6.738$ & $11.464 \pm 6.724$ & $-.357 \pm 4.506$ & $5.500 \pm 3.683$ \\
\hline \multicolumn{5}{|c|}{ Face Recognition ('Know') } \\
\hline Placebo & $11.041 \pm 5.974$ & $10.333 \pm 7.166$ & $-.708 \pm 6.025$ & $7.592 \pm 5.819$ \\
\hline $25 \mathrm{~g}$ Glucose & $10.344 \pm 5.386$ & $10.241 \pm 6.822$ & $-.103 \pm 4.117$ & $8.809 \pm 5.144$ \\
\hline $60 \mathrm{~g}$ Glucose & $8.142 \pm 4.743$ & $8.321 \pm 5.591$ & $.178 \pm 3.732$ & $7.208 \pm 3.821$ \\
\hline
\end{tabular}

